\title{
Geometric localisation in Möbius $\pi$ systems
}

\author{
Patrick W. Fowler ${ }^{\mathrm{a}, 1}$, Leonardus W. Jenneskens ${ }^{\mathrm{b}, *}$

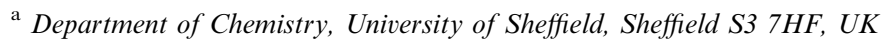 \\ b Organic Chemistry and Catalysis, Utrecht University, Padualaan 8, 3584 CH Utrecht, The Netherlands
}

Received 17 February 2006; in final form 18 June 2006

Available online 23 June 2006

\begin{abstract}
Evaluation of the eigenvalues of the bond-bond polarisability matrix for Möbius monocycles indicates that the apparent natural $\mathrm{C}_{2}$ symmetry of Möbius $\pi$ systems is not a consequence of $\pi$ distortivity but rather is due to the release of strain in the $\sigma$-skeleton.

(C) 2006 Elsevier B.V. All rights reserved.
\end{abstract}

\section{Introduction}

The Möbius band (a.k.a. Möbius strip) is a well-known object in both recreational and formal mathematics $[1,2]$. It is single-edged, single-sided and non-orientable. In the classical construction, a Möbius band is formed by joining the ends of a rectangular piece of paper, after a half twist has been introduced (Fig. 1). When viewed through the line of the join in the paper, the physical model shows instantaneous $\mathrm{C}_{2}$ symmetry, but as manipulation of the paper model easily reveals, the $C_{2}$ axis can be rotated through a full circle, so that any two antipodal points on the central circular line can in turn become the poles of a binary rotation $(R)$. The full symmetry group of the band is therefore a direct product of $\mathrm{C}_{2}$ and cyclic translation $(S)$ groups, and is isomorphic to $\mathrm{D}_{\infty}\left(\mathrm{D}_{2 \mathrm{~m}}\right.$ if a cyclic structure of $\mathrm{m}$ translationally equivalent units is embedded in the band [3]).

Realisation of the Möbius topology in molecular form has long been a target of synthetic chemistry (Fig. 2) [4], but in all reported examples, experimental or computergenerated [5-12], the object turns out to have fixed $\mathrm{C}_{2}$ geometry. Why do molecular Möbius strips lock into $\mathrm{C}_{2}$ symmetry? Why does the free motion of the $\mathrm{D}_{\infty}$ mathematical ideal not survive as an ultra-low-energy mode of the $\mathrm{C}_{2}$

\footnotetext{
* Corresponding author. Fax: +31 302534533.

E-mail addresses: P.W.Fowler@sheffield.ac.uk (P.W. Fowler), 1.w. jenneskens@chem.uu.nl (L.W. Jenneskens).

${ }^{1}$ Fax: +44 1142229346.
}

structure? The present Letter suggests that this configuration locking is not caused by $\pi$ distortivity [13-19]. It is found that the natural symmetry of a Möbius $\pi$ system is $\mathrm{C}_{2}$, for the same reason, i.e., release of strain in the $\sigma$-skeleton, that the symmetry of 1,3,5,7-cyclooctatetraene (COT) is $D_{2 d}[20]$ rather than $D_{4 h}$.

\section{Results and discussion}

For planar and spherical systems, Hückel $\pi$ energies and orbitals are obtained from the Hamiltonian matrix $\mathbf{H}=\alpha \mathbf{1}+\beta \mathbf{A}$, i.e., as eigenvalues and eigenvectors of the adjacency matrix $\mathbf{A}$ of the carbon skeleton. An identical spectrum of eigenvalues (in appropriate reduced units) is found for an untwisted network of atoms interacting through $\sigma$ bonds. In Möbius $\sigma$ systems, diagonalisation of $\mathbf{A}$ again yields Hückel energies, but for a Möbius $\pi$ system, the matrix to be diagonalised is not $\mathbf{A}$ but $\mathbf{A}^{\prime}$. In the weighted adjacency matrix $\mathbf{A}^{\prime}$, all edges crossing the 'seam' of the Möbius band carry a factor of -1 to take account of the effect of the twist on the overlap of neighbouring $p_{\pi}$ orbitals (Fig. 3) [21,22]. Eigenvalue spectra of Möbius $\sigma$ and $\pi$ systems are thus in general different. In fact, as the Möbius band is doubly covered by the cylinder (in the sense that identification of antipodal points on a cylinder produces a set of points with the topology of the band [2]), the eigenvalue spectra of $\sigma$ and $\pi$ systems for a fixed Möbius carbon skeleton form complementary halves of the spectrum of a centrosymmetric graph with twice as 
(a)

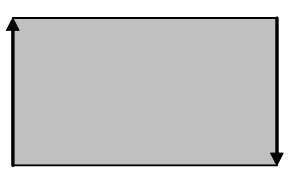

(b)

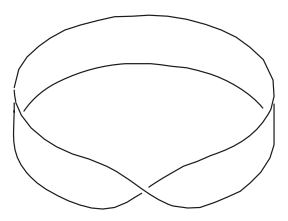

(c)
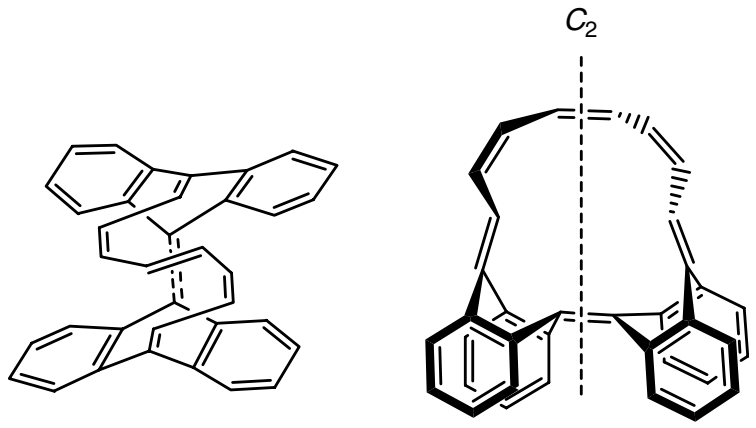

Fig. 2. A Möbius [16]-annulene with $\mathrm{C}_{2}$ symmetry $[4,12]$.

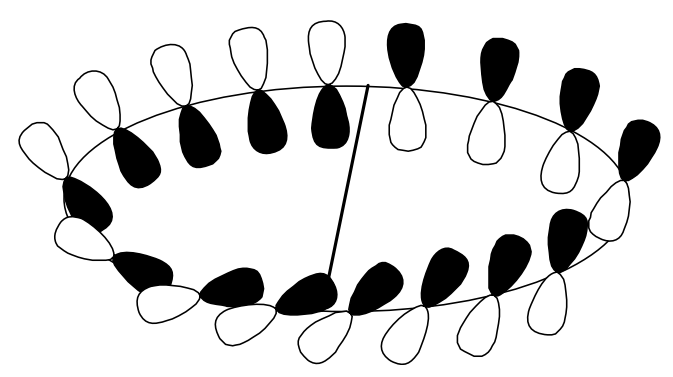

Fig. 3. Unavoidable phase interruption in a Möbius annulene. For a cycle of carbon atoms embedded on the central circle of a Mobius band, overlap of neighbouring $\mathrm{p}_{\pi}$ orbitals has a uniform sign at all positions but at one pole of the instantaneous $C_{2}$ axis of the band.

many vertices, a graph that can be embedded on the cylinder [23]. Gerade eigenvectors of the cylindrical graph give $\sigma$, ungerade $\pi$, solutions for the smaller Möbius graph. Rules for stability of $4 n+2$ and $4 n$ cycles reverse between
Hückel and Möbius systems, and the mnemonic constructions such as the Frost-Musulin [24] diagram for the eigenvalue spectra need modification, but the double-cover property allows both Hückel and Möbius cycles to be included in a single diagram for the cycle with double the number of vertices [23].

A link between geometric and electronic structure is made through the theory of $\pi$ distortivity [13-16]. In one persuasive view, $\pi$ electron delocalisation in a conjugated system is a consequence rather than a cause of high molecular symmetry. The claim for a Hückel $[4 n+2]$-annulene is that, whereas the $\sigma$ bonds of a cyclic array have a strong energetic motive for the preservation of an equilateral geometric structure, the $\pi$ electrons are inherently distortive, since $\pi$ stabilisation energy is always increased by introduction of bond alternation to localise and strengthen some $\pi$ bonds at the expense of others. A quantitative tool for the description of such distortions is the bond-polarisability matrix $\pi$ [13], the entries of which are the derivatives $\left(\partial p_{\mathrm{rs}} / \partial \beta_{\mathrm{tu}}\right)$ of the mobile $\pi$ bond order $p$ of one bond with respect to changes in the Hückel resonance parameter $\beta$ of another, and hence, apart from scaling, the $\left(\partial p_{\mathrm{rs}} / \partial \beta_{\mathrm{tu}}\right)$ are second derivatives of $\pi$ energy with respect to $\sigma$ bond lengths.

Diagonalisation of $\pi$ predicts the independent directions of distortion of the $\sigma$ framework that would favour $\pi$-electron delocalisation. In particular, when the magnitude of the maximum eigenvalue $\left|\lambda_{\max }^{\pi}\right|$ exceeds $\sim 1.8 \beta^{-1}$ for a conventional Hückel system, it is expected that actual geometric distortion will occur [13]. Thresholds for distortion of annulenes, polyenes, and other Hückel $\pi$ systems accord qualitatively with data from more sophisticated calculations and from experiment, e.g., benzene, with $\left|\lambda_{\max }^{\pi}\right|=$ $1 \beta^{-1}$ is, of course, undistorted, whereas bicyclic pentalene, with $\left|\lambda_{\max }^{\pi}\right|=2.357 \beta^{-1}$ is predicted by ab initio calculations to undergo $\mathrm{D}_{2 \mathrm{~h}} \rightarrow \mathrm{C}_{2 \mathrm{~h}}$ distortion [25].

Heilbronner gave a heuristic account [14] of this unrealised tendency to $\pi$ distortivity in benzene, from which a simple rule-of-thumb for prediction of the inherently most distortive mode of a Hückel $\pi$ system has been devised. The Heilbronner vectors [16] of a conventional $\pi$ system are sets of changes $\delta \beta_{\text {tu }}$ (which imply changes in bond lengths) such that the sum $\Sigma_{\mathrm{t}} \delta \beta_{\mathrm{tu}}$ vanishes when taken over all bonds tu radiating from a common vertex t, i.e., $\Sigma_{\mathrm{t}} A_{\mathrm{tu}} \delta \beta_{\mathrm{tu}}=0$. The space spanned by these vectors is easily identified, by analogy with the graphical construction of non-bonding orbitals, and it is typically found to contain a match to the most distortive eigenvector(s) of $\pi$. This association is easily rationalized in terms of the alternating character of entries in $\pi$ along a series of elements $\pi_{\mathrm{rs}, \mathrm{tu}}$ associated with the bonds tu in a conjugated pathway starting from a given bond rs. Thus, for Hückel annulenes of even size, the $\pi$ eigenvector of maximum $\left|\lambda^{\pi}\right|$ is entirely determined by symmetry. It is exactly the sole Heilbronner vector and is the bond alternation (Kekulé) mode associated with in-plane first-order Jahn-Teller distortion in the 
case of $[4 n]$-annulenes. For $[4 n+2]$-annulenes the series $\left|\lambda_{\max }^{\pi}\right| \beta^{-1}=1.0000,1.3416,1.5602,1.7219,1.8506,1.9575$, $2.0489,2.1288, \ldots$ for $n=1-8$ predicts loss of $\mathrm{D}_{(4 n+2) \mathrm{h}}$ symmetry by the second-order mechanism at $\sim 22$ carbon centres, coinciding with ab initio SCF estimates for the distortion threshold and falling somewhat below the DFT estimate of 30 [17-19]. For pentalene, the vertexsum requirement imposes loss of mirror symmetry in the Heilbronner vector, which indeed leads from ideal $D_{2 h}$ down to the $C_{2 h}$ point group and has entries $\delta \beta_{\text {tu }}$ that closely match the maximal eigenvector of $\pi$ (Fig. 4).

Amongst Möbius $\pi$ systems, the 'aromatic' cycles are those with $4 n \pi$ electrons [21], and $\pi$ can be calculated with the usual second-order perturbation expression, but based on the eigenvectors of $\mathbf{A}^{\prime}$ rather than $\mathbf{A}$. Maximum eigenvalues, $\left|\lambda_{\max }^{\pi}\right|$, for Möbius [4n]- and Hückel $[4 n+2]$-annulenes fall on the same curve (Fig. 5), and predict distortion at a similar threshold, if we assume the same $\beta$ value for both conventional and Möbius annulenes [21]. It may be argued that $\beta$ should be reduced by a factor of $\cos (\pi / 4 n)$ in the Möbius annulene, giving a reduction of $8 \%$ for $4 n=8$ and less than $1 \%$ for $4 n=20$. The most dis-

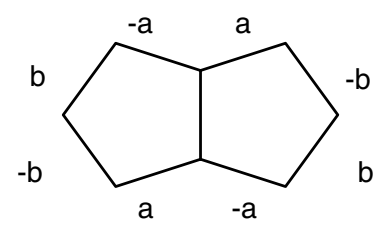

Fig. 4. Match of Heilbronner vectors and direction of maximum $\pi$ distortivity. In pentalene, the unique normalised Heilbronner vector [16] has $a=b=0.3536$, indicating equal and opposite increases and decreases of the Hückel $\beta$ parameter in alternation around the molecular perimeter, with no change to the central bond. In the exact eigenvector of $\pi$ (eigenvalue $2.357 \beta^{-1}$ ), the pattern of distortion is essentially identical, with $a=0.3428$ and $b=0.3639[13,25]$.

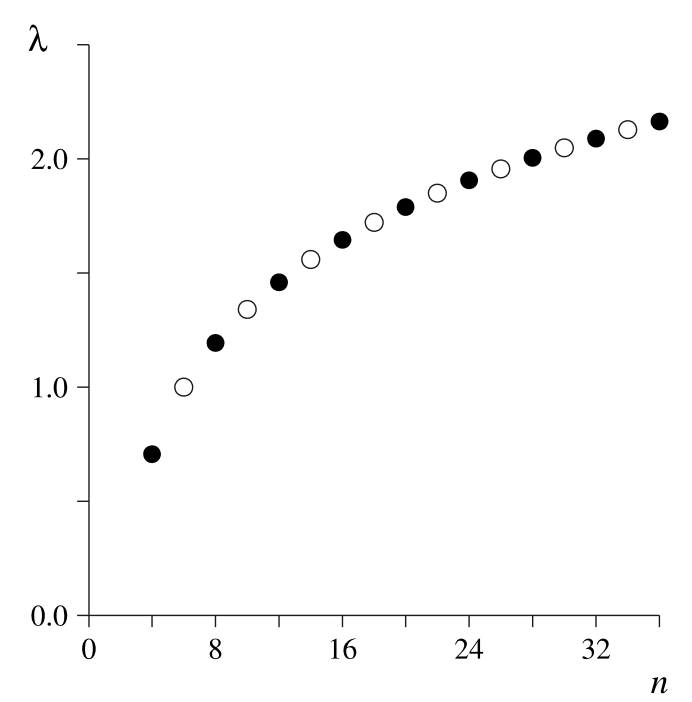

Fig. 5. Variation of maximum eigenvalue $\left|\lambda_{\max }^{\pi}\right| \beta^{-1}$ with ring size, $n$, for the aromatic Hückel $[4 n+2]$ - and Möbius [4n]-annulenes. Hückel systems are denoted by open circles ( $\circ)$ and Möbius systems by filled circles $(\bullet)$. tortive eigenvector has a characteristic form, obtainable by a simple modification of the Heilbronner construction: sets of changes $\delta \beta_{\text {tu }}$ are now found subject to a zero-sum $\Sigma_{\mathrm{t}} A_{\mathrm{tu}}^{\prime} \delta \beta_{\mathrm{tu}}=0$. Thus, effectively, we may construct the Heilbronner vector as though the system were of the normal Hückel type, and then reverse the entry $\delta \beta_{\text {tu }}$ for any bond that crosses the seam. This procedure produces a distortion vector with at most $C_{2}$ symmetry about the chosen seam position. With the usual correlation of $\beta$ with bond length, we see that the Heilbronner vector corresponds to alternant shortening and lengthening of bonds around the Möbius cycle: in a bond that does not cross the seam, a change of $A_{\mathrm{tu}}^{\prime} \beta_{\mathrm{tu}}$ to $(1+\delta) \beta_{\mathrm{tu}}$ corresponds to bond shortening; in a bond that crosses the seam it is a change of $A_{\mathrm{tu}}^{\prime} \beta_{\mathrm{tu}}$ to $(-1-\delta) \beta_{\text {tu }}$ that corresponds to bond shortening. The instantaneous molecular symmetry remains $\mathrm{C}_{2}$, but the full symmetry group falls from $\mathrm{D}_{8 n}$ to $\mathrm{D}_{4 n}$, as the translational repeat unit is now larger. The $\pi$ distortion has the same origin as the tendency of conventional large $[4 n+2]$ - and [4n]annulenes to break symmetry [17-19], though it is likely to occur sooner as a result of the extra $\sigma$ strain and the reduced $\pi$ overlap (which follows a cosine function $\cos (\pi / n)[21])$ in the Möbius band. The fact that the occupied molecular orbitals of small Mobius annulenes can be partitioned into those of a polyene and a twisted ethene fragment $[10]$ is consistent with the dominance of $\sigma$ factors. The energetic cost of such twisting can be estimated by a modified version of the Sandorfy C model [26] taking explicitly into account 1,3-interactions [27,28].

\section{Conclusion}

Qualitative chemical calculations based on the Heilbronner approach to $\pi$ distortivity indicate that the locking of configuration of Möbius-type molecules and structures is not a consequence of any special features of their $\pi$ electronic structure, but should rather be ascribed to steric effects within their $\sigma$ systems.

\section{Acknowledgement}

P.W.F. thanks the Royal Society/Wolfson Research Merit Award Scheme for partial financial support.

\section{References}

[1] S. Barr, Experiments in Topology, Dover Publications Inc., New York, 1992.

[2] J. Stillwell, Classical Topology and Combinatorial Group Theory, second edn., Springer, New York, 1993.

[3] M.A. Armstrong, Groups and Symmetry, Springer Verlag, New York, 1998

[4] D. Ajami, O. Oeckler, R. Herges, Nature 426 (2003) 819.

[5] M. Mauksch, V. Gogonea, P.v.R. Schleyer, Angew. Chem., Int. Ed. 37 (1998) 2395.

[6] S. Martin-Santamaria, B. Lavan, H.S. Rzepa, J. Chem. Soc. Perkin Trans. 2 (2000) 1415.

[7] S. Martin-Santamaria, H.S. Rzepa, J. Chem. Soc. Perkin Trans. 2 (2000) 2372. 
[8] D. Hall, H.S. Rzepa, Org. Biomol. Chem. 1 (2003) 182.

[9] S. Martin-Santamaria, H.S. Rzepa, J. Chem. Soc. Perkin Trans. 2 (2000) 2378.

[10] R.W.A. Havenith, J.H. van Lenthe, L.W. Jenneskens, Int. J. Quant. Chem. 85 (2001) 52.

[11] C. Castro, C.M. Isborn, W.L. Karney, M. Mauksch, P.v.R. Schleyer, Org. Lett. 4 (2002) 3431.

[12] C. Castro, Z. Chen, C.S. Wannere, H. Jiao, W.L. Karney, M. Mauksch, R. Putcha, N.J.R. van Eikema Hommes, P.v.R. Schleyer, J. Am. Chem. Soc. 127 (2005) 2425.

[13] G. Binsch, E. Heilbronner, J.N. Murrell, Mol. Phys. 11 (1966) 305.

[14] E. Heilbronner, J. Chem. Educ. 66 (1989) 471.

[15] S. Shaik, A. Shurki, D. Danovich, P. Hiberty, Chem. Rev. 101 (2001) 1501.

[16] P.W. Fowler, A. Rassat, Phys. Chem. Chem. Phys. 4 (2002) 1105.
[17] C.S. Wannere, P.v.R. Schleyer, Org. Lett. 5 (2003) 865.

[18] See also: C.S. Wannere, D. Moran, N.L. Allinger, B.A. Hess Jr, L.J. Schaad, P.v.R. Schleyer, Org. Lett. 5 (2003) 2983.

[19] A. Soncini, P.W. Fowler, L.W. Jenneskens, Phys Chem. Chem. Phys. 6 (2004) 277.

[20] For a review: F.G. Klärner, Angew. Chem., Int. Ed. Engl. 40 (2001) 3977.

[21] E. Heilbronner, Tetrahedron Lett. 29 (1969) 1923.

[22] H.E. Zimmermann, Acc. Chem. Res. 4 (1971) 272.

[23] P.W. Fowler, Phys. Chem. Chem. Phys. 4 (2002) 2878.

[24] A.A. Frost, B. Musulin, J. Chem. Phys. 21 (1953) 572.

[25] E. Heilbronner, S. Shaik, Helv. Chim. Acta 75 (1992) 539.

[26] C. Sandorfy, Can. J. Chem. 33 (1955) 1337.

[27] M.J.S. Dewar, J. Am. Chem. Soc. 106 (1984) 669.

[28] T. Schepers, J. Michl, J. Phys. Org. Chem. 15 (2002) 490. 